\title{
Investigation of the low-energy kaons hadronic interactions in light nuclei by AMADEUS
}

\author{
A. Scordo ${ }^{1, \star}$, M. Cargnelli ${ }^{2}$, C. Curceanu ${ }^{1}$, L. Fabbietti ${ }^{3,4}$, J. Marton $^{2}$, K. Piscicchia ${ }^{1,5}$, D. Sirghi ${ }^{1}$, \\ I. Tucakovic ${ }^{1}$, O. Vazquez Doce ${ }^{3,4}$, S. Wycech ${ }^{6}$, J. Zmeskal $^{2}$, G. Mandaglio ${ }^{7,8}$, M. Martini $^{1,9}$, and \\ P. Moskal ${ }^{10}$ \\ ${ }^{1}$ INFN, Laboratori Nazionali di Frascati, 00044 Frascati, Italy \\ ${ }^{2}$ Stefan-Meyer-Institut für Subatomare Physik, 1090 Wien, Austria \\ ${ }^{3}$ Excellence Cluster 'Origin and Structure of the Universe', 85748 Garching, Germany \\ ${ }^{4}$ Physik Department E12, Technische Universität München, 85748 Garching, Germany \\ ${ }^{5}$ Museo Storico della Fisica e Centro Studi e Ricerche Enrico Fermi, Italy \\ ${ }^{6}$ National Centre for Nuclear Research, 00681 Warsaw, Poland \\ ${ }^{7}$ Dipartimento M.I.F.T. dell'Università di Messina, 98166 Messina, Italy \\ ${ }^{8}$ INFN Gruppo collegato di Messina, 98166 Messina, Italy \\ ${ }^{9}$ Dipartimento di Scienze e Tecnologie applicate, Università 'Guglielmo Marconi', 00193 Roma, Italy \\ ${ }^{10}$ Institute of Physics, Jagiellonian University, 30-059 Cracow, Poland
}

\begin{abstract}
The AMADEUS experiment deals with the investigation of the low-energy kaon-nuclei hadronic interaction at the DAФNE collider at LNF-INFN. This study is fundamental to solve longstanding questions concerning interactions of strange quarks in the non-perturbative QCD. AMADEUS step 0 consisted in the reanalysis of the 2004/2005 KLOE data, exploiting $K^{-}$absorptions in $\mathrm{H},{ }^{4} \mathrm{He},{ }^{9} \mathrm{Be}$ and ${ }^{12} \mathrm{C}$, leading to the first invariant mass spectroscopy study with very low momentum $(100 \mathrm{MeV} / \mathrm{c})$ in-flight $K^{-}$ captures. In this paper, we present an overview of the analysis strategy, with particular emphasis on the results obtained in the analyses of the events with correlated $\Sigma^{0}$ and $p$.
\end{abstract}

\section{Introduction}

The AMADEUS experiment [1] studies the low-energy interactions of the negatively charged kaons with light nuclei. Such type of physics, important for the understanding of the non-perturbative QCD in the strangeness sector, has an important impact on hadron and nuclear physics and astrophysics. In this context, useful information can be obtained from the strength of the $K^{-}$binding in nuclei. The study of the $K^{-}$-nucleus interaction at low energies is of interest for quantifying the meson-baryon potential with strange content [2], and for its impact on models describing the structure of neutron stars (NS) [3], especially after the recent observations of heavy NS [5]. One of the most useful methodes to acquire information on the strength of the $\mathrm{KN}$ interaction is represented by the investigation of the absorption of the $\mathrm{K}^{-}$from one or more nucleons. Since one or more nucleons could be kept together by the strong attractive interaction between antikaons and nucleons, the formation of the so-called kaonic

^e-mail: alessandro.scordo@lnf.infn.it 
bound states, as $p p K^{-}$or $p p n K^{-}$, might occur. The observation of such states and the measurement of their binding energies and widths would provide a measurement of the $K$-nucleon interaction in vacuum, as an important reference for the investigation of the in-medium properties of kaons. For the di-baryonic kaonic bound state $p p K^{-}$theoretical predictions deliver a wide range of binding energies and widths [6] and experimental results are contradictory [7-13]. For the search of such states in $K^{-}$ absorption experiments, the competing multi-nucleonic absorption plays a fundamental role, but the contribution of the different single- and multi-nucleon absorption components has not been evidenced yet. Absorption processes also play an important role in the understanding of kaonic atoms, where a substantial multi-nucleon component is put forward by some theoretical models [14]. Measurements of these processes have been carried out and reported in $[15,16]$. From the analysis of the KLOE data from 2004-2005, information on both the strength of the $K^{-}$binding in nuclei and the in-medium modification of the $\Sigma^{*}$ and $\Lambda^{*}$ resonances properties can be extracted by analysing, respectively, the $\Lambda / \Sigma-p, d, t$ correlated pairs and the resonances decay channels $\Lambda / \Sigma-\pi$. In this paper, we present an overview of the analysis procedure and of different ongoing analyses. Particular emphasis is put on the results of the analysis of the $\Sigma^{0} p$ final state produced in absorption processes of $K^{-}$on two or more nucleons, occurring in the entrance wall of the KLOE drift chamber (DC), and on the search for a signature of the $p p K^{-} \rightarrow \Sigma^{0} p$ kaonic bound state.

\section{AMADEUS step 0}

The AMADEUS experiment [1] studies the low-energy interactions of the negatively charged kaons with light nuclei; it is conceived to integrate the high acceptance and momentum resolution of the KLOE detector with the low momentum $K^{-}$beam of the DAФNE collider in a future dedicated setup. DAФNE [17] (Double Anular $\Phi$-factory for Nice Experiments) is a double ring $e^{+} e^{-}$collider, designed to work at the center of mass energy of the $\Phi$ particle; the $\Phi$ meson decay produces charged kaons with low momentum $(\simeq 127 \mathrm{MeV} / \mathrm{c})$ which is ideal either to stop them, or to explore the products of their low-energy nuclear absorptions. The KLOE detector [18] is centered around the interaction region of DA $\Phi N E$ and is characterised by $\mathrm{a} \simeq 4 \pi$ geometry and an acceptance of $\simeq 98 \%$; it consists of a large cylindrical Drift Chamber (DC) and a fine sampling lead-scintillating fibers calorimeter, all immersed in the axially simmetric magnetic field with intensity of $0.52 \mathrm{~T}$, provided by a superconducting solenoid. The DC entrance wall composition is $750 \mu \mathrm{m}$ of carbon fibre and $150 \mu \mathrm{m}$ of aluminum foil. Dedicated GEANT Monte Carlo simulations of the KLOE apparatus show that out of the total number of kaons interacting in the DC entrance wall, about $81 \%$ are absorbed in the carbon fibre component and remaining $19 \%$ in the aluminum foil. The KLOE DC is filled with a mixture of helium and isobutane $\left(90 \%\right.$ in volume ${ }^{4} \mathrm{He}$ and $10 \%$ in volume $\left.\mathrm{C}_{4} \mathrm{H}_{10}\right)$. As a step 0 of AMADEUS, we analysed the 2004-2005 KLOE data, for which an important contribution of in-flight $K^{-}$nuclear captures, in different nuclear targets from the KLOE materials, was evidenced and characterised, enabling to perform invariant mass spectroscopy of in-flight $K^{-}$nuclear captures [19].

\section{The particle identification procedure}

The investigation of the negatively charged kaons interactions in nuclear matter is performed through the reconstruction of hyperon-pion and hyperon-nucleon/nucleus correlated pairs productions, following the $K^{-}$absorptions in $\mathrm{H},{ }^{4} \mathrm{He},{ }^{9} \mathrm{Be}$ and ${ }^{12} \mathrm{C}$. The investigation of the $K^{-}$multi-nucleon absorptions and the properties of possible antikaon multi-nucleon bound states proceeds through the analyses of the $\Lambda / \Sigma-p, d, t$, correlations; this last channel is, in particular, extremely promising for the search and characterisation in different nuclear targets of the extremely rare four nucleon absorption process. The 
search for the $\Lambda(1405)$ is performed through its decay into $\Sigma^{0} \pi^{0}$ (purely isospin $\mathrm{I}=0$ ) and $\Sigma^{+} \pi^{-}$. Given the excellent momentum resolution for charged particles, also the analysis of the $\Lambda \pi^{-}$(isospin $\mathrm{I}=1$ ) production, both from direct formation process and from internal conversion of a primary produced $\Sigma$ hyperon $\left(\Sigma N \rightarrow \Lambda N^{\prime}\right)$ is presently ongoing. The aim is to measure, for the first time, the module of the non-resonant transition amplitude (compared with the resonant $\Sigma^{*-}$ ) below threshold. The presence of a hyperon always represents the signature of a $K^{-}$hadronic interaction inside the KLOE setup materials. All the analyses introduced before start with the identification of a $\Lambda(1116)$, through the reconstruction of the $\Lambda \rightarrow p+\pi^{-}(\mathrm{BR}=63.9 \pm 0.5 \%)$ decay vertex. The reconstructed $M_{p \pi^{-}}$invariant mass shows a mean value of $1115.753 \pm 0.002 \mathrm{MeV} / \mathrm{c}^{2}$, with a resolution of $\sigma=0.5 \mathrm{MeV} / \mathrm{c}^{2}$, in agreement with the PDG value. The particle identification is done using energy information coming from the Drift Chamber wires and, only for the protons, also from the Calorimeter, while the momenta are reconstructed from the tracks collected in the DC; the final selection is performed via the two-dimensional plot of energy and momentum. A common vertex between the $\Lambda$ candidate and an additional proton or deuteron or triton track is then searched for. As an example, the obtained resolution on the radial coordinate $\left(\rho_{\Lambda p}\right)$ for the $\Lambda p$ vertex is $12 \mathrm{~mm}$, and this topological variable is used to select the $K^{-}$absorption processes inside the DC wall. The $\Sigma$ candidates are identified through their decay into $\Lambda \gamma$ or $\mathrm{p} \pi$ pairs. The photon selection is carried out via its identification in the EMC. Photon candidates are selected by applying a cut on the difference between the EMC time measurement and the expected time of arrival of the photon within $-1.2<t<1.8 \mathrm{~ns}$. Using the time and energy information from the electromagnetic calorimeter, $\pi^{0}$ particles can be reconstructed through their $\gamma \gamma$ decay. In order to select $K^{-}$interactions in the various targets, cuts on the radial position $\left(\rho_{\Lambda}\right)$ of the $\Lambda$ decay vertex are optimised in order to separate the two samples of $K^{-}$absorption events occurring in the DC wall and the DC gas: $\rho_{\Lambda}=25 \pm 1.2 \mathrm{~cm}$ and $\rho_{\Lambda}>33 \mathrm{~cm}$, respectively. The $\rho_{\Lambda}$ limits were set based on MC simulations and a study of the $\Lambda$ decay path. In particular, the $\rho_{\Lambda}=25 \pm 1.2 \mathrm{~cm}$ cut guarantees, for the first sample, a contamination of $K^{-}$interactions in gas as low as $\left(5.5_{-1.8}^{+1.3 \%} \%\right.$. More details on the particle identification procedure are given in [20]

\section{$4 K^{-}$absorption on two nucleons in the $\Sigma^{0} p$ final state}

The first result we report on is the search for $K^{-}$absorption on two nucleons in the $\Sigma^{0} p$ final state. The analysed data corresponds to a total integrated luminosity of $1.74 \mathrm{fb}^{-1}$ collected in 2004-2005 with the KLOE detector. The number of stopped $K^{-}$in the DC entrance wall is calculated by combining the experimental $\mathrm{K}^{+}$tagging efficiency, the luminosity information and a Monte Carlo simulation to determine the rate of $K^{-}$stopped in the DC wall. The decay nearly at rest of the $\Phi$ meson allows to tag $K^{-}$events by the identification of a $K^{+}$track in the opposite hemisphere of the DC. The total number of stopped $K^{-}$obtained in this way is equal to $(3.25 \pm 0.06) \times 10^{8}$. This value is used to normalise the measured yields of the different absorption processes. The following kinematic distributions are used simultaneously in a global fit to extract the contributions of the different absorption processes: the $\Sigma^{0} p$ invariant mass, the relative angle of the $\Sigma^{0}$ and proton in the laboratory system $\cos \left(\theta_{\Sigma^{0} p}\right)$, the $\Sigma^{0}$ and the proton momenta. The processes that are taken into account in the fit of the experimental data are: (1) $K^{-} A \rightarrow \Sigma^{0}-(\pi) p_{\text {spec }}\left(A^{\prime}\right)$, (2) $K^{-} p p \rightarrow \Sigma^{0} p$ (2NA), (3) $K^{-} p p n \rightarrow \Sigma^{0} p n$ (3NA), (4) $K^{-}$ppnn $\rightarrow \Sigma^{0}$ pnn (4NA). This list includes the $K^{-}$absorption on two nucleons (2NA) with (2NAFSI) and without (2NA-QF) final state interaction for the $\Sigma^{0} p$ state and processes involving more than two nucleons in the initial state (3NA, 4NA). For all the details on how the distributions of the different contributions are modelled and sampled please refer to [20]. Panels (a)-(d) in Fig. 1 show the experimental distributions for the $\Sigma^{0} p$ invariant mass, the $\cos \left(\theta_{\Sigma^{0} p}\right)$, and the $\Sigma^{0}$ and proton momenta, together with the fit results. The black points represent the experimental data after the subtraction 

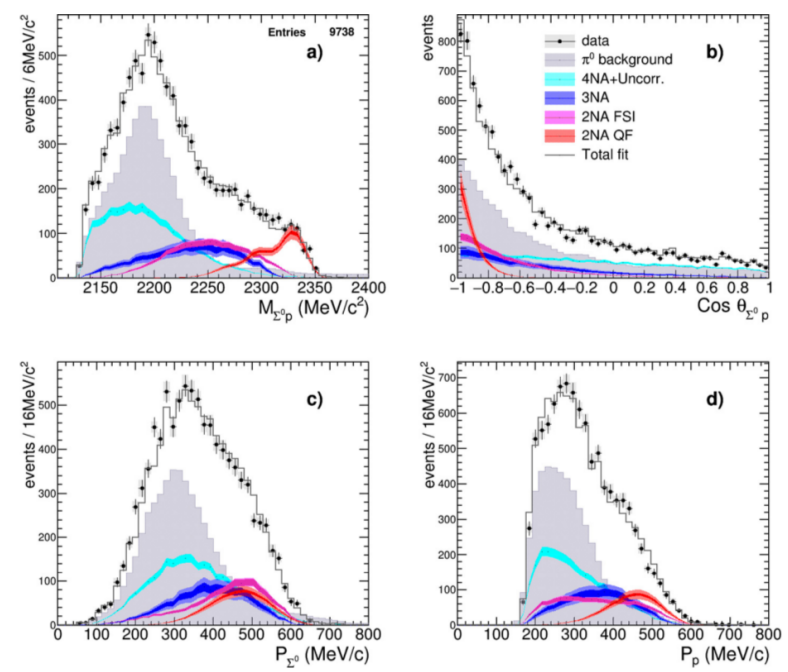

Figure 1. (Colour online.) Experimental distributions of the $\Sigma^{0} p$ invariant mass, $\cos \left(\theta_{\Sigma^{0}}\right), \Sigma^{0}$ momentum and proton momentum together with the results of the global fit. The experimental data after the subtraction of the machine background are shown by the black circles, the systematic errors are represented by the boxes and the coloured histograms correspond to the fitted signal distributions where the light-coloured bands show the fit errors and the darker bands represent the symmetrised systematic errors. The gray line shows the total fit distributions (see text for details).

of the machine background with the systematic errors shown by boxes. The gray-filled histogram represents the $\pi^{0} p$ background. The cyan distributions show the sum of the 4NA simulation together with the uncorrelated production of the $\Sigma^{0} p$ final state. The blue distribution represents the 3NA and the magenta histogram the 2NA-FSI. The red distribution shows the 2NA-QF. The gray line is the resulting total fit. For each fitted distribution the light error band corresponds to the statistical error resulting from the fit, while the darker band visualises the symmetrised systematic error (refer to [20] for more details). The fit results lead to the first measurements of the genuine 2NA-QF for the final state $\Sigma^{0} p$ in reactions of stopped $K^{-}$on targets of ${ }^{12} \mathrm{C}$ and ${ }^{27} \mathrm{Al}$. This contribution is found to be about $9 \%$ of the total absorption cross-section including 2NA, 3NA and 4NA processes with also the contribution of the uncorrelated background leading to a $\Sigma^{0} p$ final state taken into account. The last step of the analysis consists in the search for the $p p K^{-}$bound state produced in $K^{-}$interactions with nuclear targets, decaying into a $\Sigma^{0} p$ pair. The $p p K^{-}$are simulated similarly to the 2NA-QF process but sampling the mass of the $p p K^{-}$state with a Breit-Wigner distribution, rather than the Fermi momenta of the two nucleons in the initial state. The event kinematic is implemented by imposing the momentum conservation of the $p p K^{-}$-residual nucleus system. Different values for the binding energy and width varying within $15-75 \mathrm{MeV} / \mathrm{c}^{2}$ and $30-70 \mathrm{MeV} / \mathrm{c}^{2}$ in steps of 15 and $20 \mathrm{MeV} / \mathrm{c}^{2}$ , respectively, are tested. This range is selected according to theoretical predictions [6] and taking into account the experimental resolution. The global fit is repeated adding the $p p K^{-}$state to the processes 1-4. The best fit ( $\chi^{2} / \mathrm{ndf}=0$. 807) is obtained for a $p p K^{-}$candidate with a binding energy of $45 \mathrm{MeV} / \mathrm{c}^{2}$ and a width of $30 \mathrm{MeV} / \mathrm{c}^{2}$, respectively. The resulting yield normalised to the number of stopped $K^{-}$is $p p K^{-} / K_{\text {stop }}^{-}=\left(0.044 \pm 0.009\right.$ stat $\left.t_{-0.005}^{+0.004} s y s t\right) \times 10^{-2}$. Considering also that the improvement of the $\chi^{2}$ is only marginal, an F-Test is carried out to compare the two models: with 
and without $p p K^{-}$bound state. This test consists in evaluating the statistical significance of the model with the $p p K^{-}$, accounting for the additional fit parameter, by comparing the residuals and numbers of degrees of freedom of two models. The results of this F-Test shows that even the best fit corresponds to a p-value equal to 0.25 and hence to a significance of $1 \sigma$ (see [20] for more details). This implies that although the fit favours the presence of an additional component that can be parametrised with a Breit-Wigner distribution with a certain mass and width, its significance is not sufficient to claim the observation of the bound state.

\section{$5 \mathrm{KN}$ interaction studies in other channels}

\subsection{NA process in $\Lambda t$ events}

The experimental search for a $\Lambda$ and a triton in the final state of $K^{-}$induced interactions on helium, representing the golden channel for the study of the 4NA process, was published by two experiments $[21,22]$ but the contribution from $4 \mathrm{NA} \mathrm{K}^{-}$capture was not clearly identified. Analysing the KLOE 2004-2005 data, the highest statistics ever of correlated $\Lambda t$ pairs from $K^{-}$nuclear absorption in ${ }^{4} \mathrm{He}$ ( 150 events) can be obtained; in addition, as shown in Fig. 2 , for the first time the 4NA process events can be kinematically evidenced. Following energy and momentum conservations, the events related to

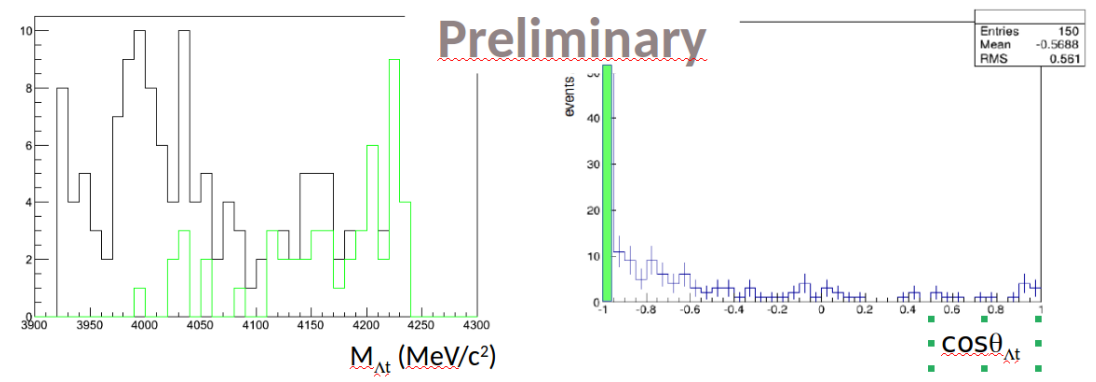

Figure 2. (Colour online.) $\Lambda t$ invariant mass and $\cos \theta_{\Lambda t}$; in green, events for $\cos \theta_{\Lambda t}<-0.95$ are shown.

the simultaneous absorption of a $K^{-}$on the 4 nucleons of the ${ }^{4} \mathrm{He}$ are characterised by an almost backto-back configuration. The simultaneous fit of $\Lambda t$ invariant mass, momentum and angular correlation, presently undergoing, will enabled to isolate for the first time the contribution of the $\mathrm{K}^{-}+{ }^{4} \mathrm{He} \rightarrow \Lambda t$ 4NA process.

\subsection{The key role of the $\Lambda(1405)$ and its detection through the $\Sigma^{+} \pi^{-}$decay}

The full understanding of the $\Lambda(1405)$ structure is one of the hottest topics in the low energy KN interactions domain. In order to understand its nature, new measurements involving non-zero momentum $K^{-}$absorptions are mandatory [24]. Analysing the events occurring in the DC entrance wall it is possible to identify a subsample of $K^{-}$interacting on the hydrogen and the carbon contained in it. These events provide, indeed, the proof of the presence in the 2002-2005 KLOE data of both in-flight and at-rest interactions. To confirm this hypothesis, in 2012 a dedicated run with a pure carbon target was performed with the KLOE detector at DAФNE; in this configuration, all the kaons are stopped in the carbon target, so only at-rest events can be found. Fig. 3 shows the $\Sigma^{+} \pi^{-}$invariant mass spectra for 
the 2004-2005 (left) compared to the 2012 (right) data. In 2004-2005 data, in-flight (green), at-rest (violet) and total (blue) events are shown together with interactions with hydrogen atoms present in the DC entrance wall. In the right panel, 2012 data (blue) are compared with the 2004-2005 (black) data to show the difference due to in-flight events [24]. These spectra enable unique possibility to

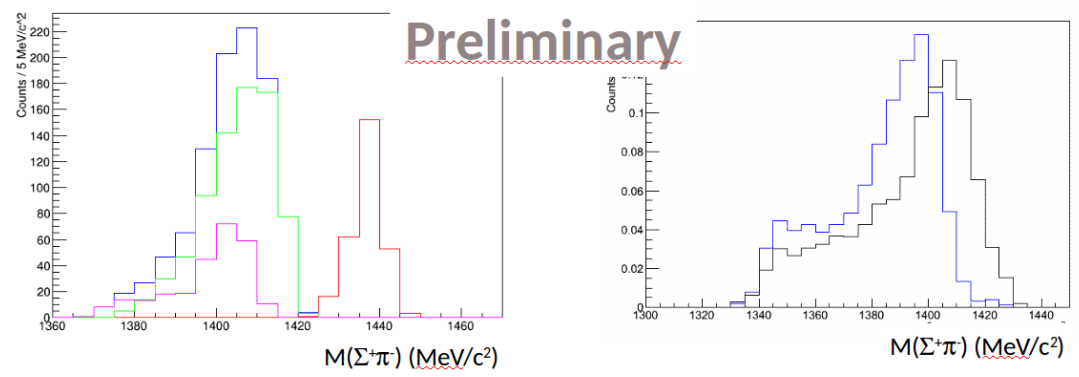

Figure 3. (Colour online.) Left panel: 2004-2005 data in-flight (green), at-rest (violet) and total (blue) events are shown together with events occurring on hydrogen atoms also present in the DC entrance wall. Right panel, 2012 data (blue) are compared with the 2004-2005 (black) data to show the difference due to in-flight events.

explore higher invariant mass region, not accessible untill now in experiments using kaons.

In addition to the analysis shown above, other interesting processes can be studied. Multi-nucleon absorption can be also studied in the $\Sigma^{0} t$ channel. Analysis on $\Lambda / \Sigma p, d$, and $\Sigma t$ channels are also ongoing. The $\Lambda(1405)$ resonance can be studied in its pure $\mathrm{I}=0 \Sigma^{0} \pi^{0}$ decay channel, whilst the ratio between the resonant and non-resonant contribution to the $\Sigma \pi$ channel can be studied starting from the pure $\mathrm{I}=1 \Lambda \pi^{-}$decay of the $\Sigma^{*}(1385)$ [25].

\section{Conclusions and outlook}

The broad experimental program of AMADEUS, dealing with the non-perturbative QCD in the strangeness sector, is supported by the quest for high precision and statistics measurements, able to set more stringent constraints on the existing theoretical models. We demonstrated the capabilities of performing high-quality research on nuclear interactions of strange particles, taking advantage of the KLOE detector and the DAФNE factory. Our investigations, presently spread on a wide spectrum of physical processes like $\Lambda / \Sigma-p, d, t$ correlations and $\Lambda^{*}(1405) / \Sigma^{*}(1385)$ properties investigations, represent the most ambitious and systematic effort in the field of low energy KN interactions. In particular, after general considerations on the analysis procedures and a brief overview on the $K^{-}+{ }^{4} \mathrm{He} \rightarrow \Lambda t$ and $K^{-} p \rightarrow \Sigma^{+} \pi^{-}$analyses, in this report we have presented the results of the $K^{-}$ absorption processes leading to the $\Sigma^{0} p$ final state measured with the KLOE detector. The absorption on two nucleons without final state interaction (2NA-QF) is isolated and its yield normalised to the number of absorbed $K^{-}$is presented. The possibility to accomodate in the final spectra a $p p K^{-}$bound state decaying into a $\Sigma^{0} p$ pair has been also carried out and the best value of the total reduced $\chi^{2}$ is achieved for the hypothesis of a $p p K^{-}$with a binding energy of $45 \mathrm{MeV} / \mathrm{c}^{2}$ and a width of $30 \mathrm{MeV} / \mathrm{c}^{2}$. A F-test is conducted to compare the simulation models with and without the $p p K^{-}$signal and to extract the significance of the result. A significance of only $1 \sigma$ is obtained for the $p p K^{-}$yield result. For the future we plan to realise a dedicated AMADEUS setup, where to explore more in details the various processes with a broad range of dedicated targets. 


\section{Acknowledgement}

We acknowledge the KLOE Collaboration for their support and for having provided us the data and the tools to perform the analysis presented in this paper.

\section{References}

[1] The AMADEUS collaboration, LNF preprint, LNF/9607/24(IR).

AMADEUS Letter of Intent:

http://www.lnf.infn.it/esperimenti/siddharta/LOI_AMADEUS_March2006.pdf

[2] D. Cabrera et al., Phys. Rev. C 90, 0552017 (2014)

[3] A. E. Nelson et al., Phys. Lett. B 192, 193 (1987)

[4] M. Bazzi et al. (SIDDHARTA Coll.), Phys. Lett. B 704, 113 (2011)

[5] P. Demorest et al., Nature 467, 1081 (2010)

[6] T. Yamazaki, et al., Phys. Rev. C 76045201 (2007)

A. Doté, et al., Phys. Rev. C 79014003 (2009)

S. Wycech, et al., Phys. Rev. C 79014001 (2009)

N. Barnea, et al., Phys. Lett. B 712132 (2012)

N.V. Shevchenko, et al., Phys. Rev. Lett. 98082301 (2007)

Y. Ikeda, et al., Phys. Rev. C 79035201 (2009)

E. Oset, et al., Nucl. Phys. A 881127 (2012)

[7] G. Agakishiev, et al., HADES Coll., Phys. Lett. B 742242 (2015)

[8] M. Agnello, et al. (FINUDA Coll.), Phys. Rev. Lett. 94212303 (2005)

[9] T. Yamazaki, et al., Phys. Rev. Lett. 104132502 (2010)

[10] Y. Ichikawa, et al., Prog. Theor. Exp. Phys. $021 D 01$ (2015)

[11] A. O. Tokiyasu et al., Phys.Lett. B 728, 616 (2014)

[12] L. Fabbietti et al., Nucl. Phys. A 914, 60 (2013)

[13] T. Hashimoto et al., Prog. Theor. Exp. Phys. 061D01 (2015)

[14] A. Gal, Nucl. Phys. A 914270 (2013)

[15] P. A. Katz et al., Phys. Rev. D 11267 (1970)

[16] M. Agnello, et al., Phys. Rev. C 92045204 (2015)

[17] INFN (1990) 371 p. and Frascati INFN - LNF-90-031(R) (90,rec.Jun.) 371 p

[18] F. Bossi et al. (KLOE coll.), Nuovo Cim. 31 531-623 (2008)

[19] K. Piscicchia et al. PoS Bormio2013 034 (2013)

[20] O. Vazquez Doce et al. Phys. Lett. B 758 134-139 (2016)

[21] R. Roosen, J. H. Wickens, Il Nuovo Cim. A Series 11, 66101 (1981)

[22] FINUDA collaboration, Phys. Lett. B, 669229 (2008)

[23] J. Beringer, et al., (Particle Data Group), Phys. Rev. D 86, 010001 (2012)

[24] A. Scordo, et al., PoS Bormio2014 039 (2014)

[25] K. Piscicchia, S. Wycech, Nucl. Phys. A 954, 75-93 (2016) 